\title{
PREVALÊNCIA E FATORES DE RISCO ASSOCIADOS À INCONTINÊNCIA URINÁRIA EM ACADÊMICAS DO CURSO DE FISIOTERAPIA
}

\section{PREVALENCE AND RISK FACTORS ASSOCIATED WITH URINARY INCONTINENCE IN ACADEMICS OF PHYSICAL THERAPY COURSE}

\section{Carolina Fernandes* (D), Luciana Cristina Rafael Ognibeni (D)}

UNINGÁ - Centro Universitário Ingá, Maringá, PR, Brasil.

*fisiocarolfernandes@gmail.com

\section{RESUMO}

A incontinência urinária, de etiologia multifatorial, pode ocorrer em diferentes idades e sexo, afetando de maneira adversa a qualidade de vida das pessoas que são acometidas. Dessa forma, o presente estudo teve como objetivo identificar a prevalência e os fatores de risco associados à incontinência urinária em acadêmicas do curso de Fisioterapia de uma Instituição de Ensino Superior, em Maringá - PR. Para isso, realizou-se uma pesquisa do tipo exploratória, de caráter transversal, quantitativa e descritiva, que teve como base a aplicação de questionários nas dependências da instituição. As acadêmicas receberam a Ficha para Identificação dos Fatores de Risco e o questionário International Consultation on Incontinence Questionnaire - Short Form (ICIQ-SF), para preenchimento. Os dados obtidos foram analisados estatisticamente, sendo apresentados em: médias, desvio-padrão, frequências e porcentagens. Para avaliar a associação entre variáveis foi utilizado o teste de Qui-quadrado. A amostra foi composta por 79 participantes, destas apenas $16(20,3 \%)$ apresentaram incontinência e somente a infecção urinária $(p=0,020)$ foi o fator de risco identificado neste estudo. Concluiu-se que apenas uma parcela das acadêmicas apresentou incontinência urinária e, entre os fatores de risco avaliados, somente a infecção urinária evidenciou associação com a perda involuntária de urina.

Palaviras-chave: Fatores de risco. Incontinência urinária. Infecção. Jovens.

\section{ABSTRACT}

Urinary incontinence, of multifactorial etiology, can occur at different ages and sex, adversely affecting the quality of life of people who are suffer. Thus, the present study aimed to identify the prevalence and risk factors associated with urinary incontinence in students of the Physical Therapy Course of a Higher Education Institution, in Maringá - PR. This is an exploratory, cross-sectional and descriptive quantitative research that was based on the application of questionnaires in the institution's facilities. The students received the Risk Factors Identification Form and the International Consultation on Incontinence Questionnaire - Short Form (ICIQ-SF), to be completed. The data obtained were statistically analyzed and presented as means, standard deviation, frequencies and percentages. To assess the association between variables, the Chi-square test was used. The sample consisted of 79 participants, of which only $16(20,3 \%)$ had incontinence and only urinary tract infection $(\mathrm{p}=0,020)$ was the risk factor identified in this study. We concluded that only a group of the students had urinary incontinence and, among the risk factors evaluated, only urinary infection was associated with involuntary loss of urine.

Keywords: Infection. Risk factors. Urinary incontinence. Young. 


\section{INTRODUÇÃO}

A continência urinária é denominada como a capacidade normal que um indivíduo possui para armazenar urina, tendo de forma consciente o controle sobre o tempo e lugar para realizar tal necessidade (POLDEN; MANTLE, 2002).

O trato urinário inferior é responsável pela continência urinária, sendo constituído pela bexiga e uretra. A bexiga tem como função armazenar e expelir a urina. Esse órgão é formado por três camadas de musculatura lisa que juntas compõem o músculo detrusor, responsável pela micção. Já a uretra é o canal de eliminação da urina, que possui dois esfíncteres: um interno composto por musculatura lisa e outro externo formado por músculo estriado, cujo funcionamento é voluntário (BARACHO, 2002; GROSSE; SENGLER, 2002).

A alternância entre a fase de enchimento e de micção corresponde ao ciclo da micção. Fisiologicamente na fase de enchimento ou período entre as micções, a pressão intrauretral é maior que a pressão intravesical, isso se deve à propriedade viscoelástica do músculo detrusor e ao controle neurológico. O simpático é responsável pelo relaxamento do detrusor e pela manutenção do tônus do esfíncter liso da uretra, e o somático pelo esfíncter estriado. E a fase miccional ou de esvaziamento é regulada pelo parassimpático, que provoca contração do músculo detrusor levando ao aumento da pressão intravesical e a pressão uretral é anulada pelo relaxamento do esfíncter liso e estriado, permitindo a micção (GROSSE; SENGLER, 2002; POLDEN; MANTLE, 2002).

Segundo Baracho (2002) para que exista continência é necessário que a pressão intrauretral seja maior que a intravesical, o que só acontece se o trato urinário estiver anatômica e fisiologicamente preservado.

Vale ressaltar que a manutenção da continência urinária ainda é favorecida pela musculatura que constitui o assoalho pélvico, em conjunto com a fáscia endopélvica. Além disso, esses músculos auxiliam no suporte dos órgãos pélvicos (FORTUNATO et al., 2014).

$\mathrm{Na}$ mulher a continência urinária é mantida pela estabilidade do músculo detrusor e pela manutenção do gradiente de pressão entre bexiga e uretra. Esse gradiente é dependente de dois fatores, os extrínsecos e os intrínsecos. Os fatores extrínsecos compreendem o músculo elevador do ânus e a fáscia endopélvica com seus ligamentos, lesões da fáscia e/ou suporte muscular fraco ocasionam hipermobilidade do colo vesical e uretra proximal. Nesses casos, a pressão abdominal é propagada apenas à bexiga e o gradiente de pressão com a uretra aumenta, resultando na perda de urina (MONTELLATO; BARACATI; ARAP, 2000).

Os fatores intrínsecos correspondem à musculatura lisa e estriada da parede uretral, à congestão dos vasos sanguíneos da uretra, à coaptação das dobras do epitélio de revestimento e ao tônus uretral. Essas estruturas permitem o fechamento da uretra, contudo, algumas situações podem levar a uma insuficiência esfincteriana intrínseca, como cirurgias, traumas, diminuição do nível de estrogênio e lesões neurológicas, ocasionando a incontinência urinária (MONTELLATO; BARACATI; ARAP, 2000).

A Incontinência Urinária (IU) é definida, de acordo com a International Continence Society, como perda involuntária de urina. Essa perda reflete nas questões físicas, emocionais, sexuais, sociais e econômicas, interferindo negativamente na qualidade de vida dos indivíduos acometidos (JUSTINA, 2013).

A classificação da IU se dá em três tipos: IU de esforço, de urgência e mista. A IU de esforço ocorre quando a elevação da pressão intravesical ultrapassa a pressão uretral ocasionando perda de urina através de esforços como, por exemplo, tossir, espirrar, rir e levantar pesos. Na IU de urgência a perda involuntária de urina está acompanhada do desejo imperioso de urinar e da contratura do detrusor. Já a IU mista decorre da associação dos sintomas da IU de esforço e de urgência (BARACHO, 2002; GROSSE; SENGLER, 2002).

A IU de esforço é frequentemente a mais relatada e mais comum em mulheres jovens, enquanto entre idosas prevalecem a IU mista e a de urgência (BEREK, 2012). 
Além disso, a IU é considerada como um sintoma multifatorial. Os principais fatores de risco que contribuem para o seu aparecimento são: sexo, idade, etnia, paridade, tipo de parto, menopausa, obesidade, atividade física, doenças crônicas, infecções do trato urinário recorrente, lesões neurológicas, consumo de cafeína e tabagismo (HIGA; LOPES; REIS, 2008; MOURÃO et al., 2017).

Nesse contexto, a presente pesquisa teve como objetivo avaliar a prevalência e os fatores de risco associados à incontinência urinária em acadêmicas do Curso de Fisioterapia de uma Instituição de Ensino Superior, localizada em Maringá.

\section{MATERIAL E MÉTODOS}

Este estudo foi aprovado pelo Comitê de Ética em Pesquisas Envolvendo Seres Humanos do Centro Universitário Ingá-UNINGÁ, sob o parecer de $n^{\circ} 3.324 .521$.

Trata-se de um estudo do tipo transversal, exploratório de caráter quantitativo descritivo, baseado na aplicação de questionários às acadêmicas do curso de Fisioterapia do período noturno, de uma Instituição de Ensino Superior localizada em Maringá - PR, em junho de 2019.

A participação na pesquisa obedeceu aos seguintes critérios de inclusão: sexo feminino e idade igual ou superior a 18 anos. Quanto aos critérios de exclusão foram: não preencher corretamente os questionários e não estar presente na sala de aula no dia em que o questionário foi aplicado.

As que aceitaram participar da pesquisa assinaram o Termo de Consentimento Livre e Esclarecido (TCLE), preencheram a Ficha auto estruturada de Identificação dos Fatores de Risco associados à Incontinência Urinária, e responderam ao questionário ICIQ-SF - International Consultation on Incontinence Questionnaire - Short Form, traduzido e validado para o português por Tamanini et al. (2004).

Com referência à sua composição o ICIQ-SF é composto por quatro questões que avaliam a frequência, a quantidade de urina perdida e o impacto da IU, além de oito itens relacionados às causas ou situações de IU vivenciadas pelas participantes, com base nas últimas quatro semanas da data de aplicação do questionário. O escore geral é obtido pela soma das questões 3 (frequência), 4 (quantidade) e 5 (impacto); quanto maior o escore, maior o impacto sobre a qualidade de vida, podendo variar de 0 a 21 pontos.

A aplicação dos questionários foi realizada em salas de aula, nas turmas do primeiro ao quinto ano. Antes das acadêmicas responderem aos questionários, a pesquisadora realizou uma explicação prévia a respeito do objetivo da pesquisa.

Dessa forma, as informações obtidas pelos questionários foram compiladas no programa Microsoft Office Excel (® 2010 onde foram organizadas por meio de planilhas, para análise posterior. Os resultados foram apresentados em: médias, desvio-padrão, frequências (n) e porcentagens (\%). E para verificar a associação entre variáveis foi utilizado o teste de Quiquadrado, com nível de significância estabelecido em $5 \%(p<0,05)$. Os dados foram analisados com auxílio do programa estatístico Statiscal Package for the Social Sciences (SPSS), versão 23.

\section{RESULTADOS E DISCUSSÃO}

Os questionários foram aplicados a 102 acadêmicas no total, sendo que 23 foram excluídos por não se enquadrarem nos critérios de inclusão da pesquisa e/ou por estarem preenchidos incorretamente, assim, apenas 79 questionários foram utilizados para análise dos resultados.

De acordo com os resultados obtidos na Ficha de Fatores de Risco Associados à IU, a média de idade das acadêmicas foi de 22,08 $\pm 3,6$ anos e a média do Índice de Massa Corporal (IMC) foi de $23,7 \pm 3,5 \mathrm{~kg} / \mathrm{m}^{2}$.

Todas as participantes eram não tabagistas (100\%); 60,8\% de etnia branca; $92,4 \%$ nulíparas; $31,6 \%$ das acadêmicas já tiveram de 2 a 3 episódios de infecção urinária; 83,5\% possuíam a 
menstruação regular; apenas uma apresentava Hipertensão Arterial Sistêmica (HAS); nenhuma possuía doença neurológica (100\%); 58,2\% ingeriam café; 51,9\% bebidas alcoólicas; 82,2\% suco; 51,9 chá; $68,3 \%$ ingeriam refrigerante; e 69,6\% eram sedentárias (Tabela 1).

Tabela 1 - Fatores de risco associados à incontinência urinária presentes nas acadêmicas

\begin{tabular}{|c|c|c|}
\hline \multicolumn{3}{|l|}{ Fatores } \\
\hline Idade & $\mathbf{N}$ & $\%$ \\
\hline 18 & 12 & 9,48 \\
\hline 19 & 7 & 5,53 \\
\hline 20 & 9 & 7,11 \\
\hline 21 & 15 & 11,85 \\
\hline 22 & 9 & 7,11 \\
\hline 23 & 10 & 7,90 \\
\hline 24 & 1 & 0,79 \\
\hline 25 & 2 & 1,58 \\
\hline 26 & 5 & 3,95 \\
\hline 27 & 3 & 2,37 \\
\hline 28 & 1 & 0,79 \\
\hline 30 & 1 & 0,79 \\
\hline 31 & 1 & 0,79 \\
\hline 33 & 3 & 2,37 \\
\hline \multicolumn{3}{|l|}{ Etnia } \\
\hline Branca & 48 & 60,8 \\
\hline Negra & 4 & 5 \\
\hline Parda & 27 & 34,2 \\
\hline Amarela & 0 & 0 \\
\hline \multicolumn{3}{|l|}{ Tabagismo } \\
\hline Não tabagista & 79 & 100 \\
\hline Tabagista & 0 & 0 \\
\hline \multicolumn{3}{|c|}{ Número de parto } \\
\hline Vaginal & 1 & 1,3 \\
\hline Cesária & 5 & 6,3 \\
\hline Nulípara & 73 & 92,4 \\
\hline \multicolumn{3}{|c|}{ Infecção urinária } \\
\hline $1 \mathrm{vez}$ & 23 & 29,1 \\
\hline $2-3$ vezes & 25 & 31,7 \\
\hline $4-5$ vezes & 3 & 3,8 \\
\hline$>5$ & 5 & 6,3 \\
\hline Nenhuma vez & 23 & 29,1 \\
\hline \multicolumn{3}{|l|}{ Menstruação } \\
\hline Regular & 66 & 83,5 \\
\hline Irregular & 9 & 11,4 \\
\hline Não menstrua & 4 & 5,1 \\
\hline \multicolumn{3}{|c|}{ Doença crônica } \\
\hline HAS & 1 & 1,3 \\
\hline Não possui & 78 & 98,7 \\
\hline \multicolumn{3}{|c|}{ Doença neurológica } \\
\hline Não possui & 79 & 100 \\
\hline \multicolumn{3}{|c|}{ Ingestão de líquidos } \\
\hline Água & 79 & 100 \\
\hline Café & 46 & 58,2 \\
\hline
\end{tabular}


Tabela 1, cont.

\begin{tabular}{lcc} 
Bebida alcoólica & 41 & 51,9 \\
Suco & 65 & 82,2 \\
Chá & 41 & 51,9 \\
Refrigerante & 54 & 68,3 \\
\hline Atividade Física & & \\
\hline Caminhada & 2 & 2,5 \\
Ciclismo & 2 & 2,5 \\
Corrida & 1 & 1,3 \\
Musculação & 14 & 17,7 \\
Musculação/jump & 1 & 1,3 \\
Natação & 1 & 1,3 \\
Pilates & 1 & 1,3 \\
Pilates/ciclismo & 1 & 1,3 \\
Vôlei de praia & 1 & 1,3 \\
Não pratica & 55 & 69,6 \\
\hline
\end{tabular}

Fonte: os autores.

Nesta pesquisa foi utilizado como critério de inclusão o sexo feminino, por ser predominantemente mais acometido pela IU, em relação ao sexo masculino. De acordo com Grosse e Sengler (2002) esse fato pode ser explicado por diferenças anatômicas, pois, a uretra da mulher é mais curta que a do homem e este dispõe de um sistema perineal fechado. Além disso, as forças de retenção da mulher são mais frágeis e estão submetidas a fatores que contribuem para sua redução tais como, traumas obstétricos, idade, menopausa e alterações hormonais.

Dentre os fatores de risco associados à IU, um deles é a idade. A idade avançada é considerada um fator de risco relevante, uma vez que nas mulheres, com o passar dos anos, há uma redução dos níveis de estrógeno o que leva à atrofia da musculatura uretral facilitando o aparecimento da IU, processo este que se agrava no período da menopausa, considerada outro fator de risco. Porém, vale ressaltar, que a perda involuntária de urina não é exclusiva de mulheres idosas, visto que existe também uma porcentagem de mulheres jovens que são acometidas (PATRIZZI et al., 2014; CÂNDIDO et al., 2017), o que instigou a realização deste estudo.

Segundo Leroy, Lopes e Shimo (2012) o predomínio da IU varia conforme a etnia, sendo maior nas mulheres brancas, independente da idade. E em relação aos tipos de IU, a de esforço é mais comum entre brancas e a de urgência em negras.

Em um estudo de revisão realizado por Higa, Lopes e Reis (2008) os autores demostraram que a relação da obesidade com a IU pode ser explicada pelo aumento da pressão intra-abdominal e, consequentemente, da pressão intravesical, que ocasionam uma alteração no mecanismo do trato urinário. Assim como a obesidade, considera-se que atividade física vigorosa e de alto impacto também contribua para o aumento da pressão intra-abdominal. Em contrapartida, Virtuoso, Mazo e Menezes (2011) discorrem que a atividade física pode contribuir para o aumento do volume muscular do assoalho pélvico, auxiliando no processo de contração durante a elevação da pressão intra-abdominal. No presente estudo, $69,6 \%$ das participantes eram sedentárias e a média do IMC foi de $23,7 \mathrm{~kg} / \mathrm{m}^{2}$, dado obtido pelo peso e altura coletados através da Ficha de Identificação dos Fatores de Risco. Segundo a classificação da Organização Mundial de Saúde (OMS) válida para pessoas adultas, as mesmas se encontram com o peso adequado (BRASIL, 2017).

Ademais, no estudo de Higa, Lopes e Reis (2008), os autores relataram que a prevalência de IU aumenta com o grau de paridade. Além disso, o parto vaginal associado às lesões e traumas do assoalho pélvico favorece o surgimento de IU. Quanto aos tipos de parto, tanto o parto vaginal como a cesárea demonstraram associação com a perda involuntária de urina. No presente estudo 92,4\% das acadêmicas eram nulíparas. De acordo com Santos et al. (2009) a presença de IU em 
jovens nulíparas, pode ser decorrente da fraqueza genética do tecido conjuntivo, do número reduzido de fibras musculares do assoalho pélvico ou localização mais baixa dessa região.

A IU também pode ter relação com a infecção recorrente do trato urinário. Neste estudo 56 participantes $(70,9 \%)$ já tiveram entre um ou mais de cinco episódios de infecção urinária. A infecção causada por vírus, bactérias ou fungos, provoca instabilidade do músculo detrusor e redução da pressão de fechamento da uretra, predispondo ao surgimento da IU (BARACHO, 2002; MOURÃO et al., 2017).

Apesar de que no presente estudo não tenha sido relatada, por parte das participantes, a presença de lesões neurológicas, estas possuem relação com a IU, pois patologias envolvendo o cérebro, a medula espinhal e os nervos periféricos podem alterar o funcionamento e a estrutura do esfíncter e da bexiga, além do equilíbrio vesicoesfincteriano (GROSSE; SENGLER, 2002; MCANINCH; LUE, 2014).

Entre as doenças crônicas que se associam à IU a mais relatada é o Diabetes Mellitus. Acredita-se que a hiperglicemia causa danos aos nervos autônomos da bexiga, prejudicando seu funcionamento. Neste estudo não foi mencionado por nenhuma participante presença de diabetes, apenas uma relatou como doença crônica, a HAS. Os medicamentos usados para o tratamento dessa doença, como diuréticos e drogas anti-hipertensivas, podem levar ao aumento da frequência e do desejo de urinar, predispondo o indivíduo à IU (JARDIM et al., 2011; CÂNDIDO et al., 2017).

No que concerne à ingestão de líquidos, estudos mostram que as bebidas que possuem cafeína, como chá, café e refrigerantes à base de cola, aumentam a diurese e a contratilidade da musculatura lisa do detrusor. Além disso, bebidas alcoólicas, sucos e frutas cítricas também causam excitação a esse músculo, assim, a ingestão elevada destas bebidas pode levar à perda involuntária de urina (HIGA; LOPES; REIS, 2008; GASPARETTO et al., 2011; TETTAMANTI et al., 2011).

Por fim, autores contestam a probabilidade de o tabagismo ser um fator de risco, pois os fumantes apresentam uma tosse mais vigorosa, o que ocasiona um efeito direto na bexiga e na uretra, danificando o mecanismo de contenção da urina. Além disso, alguns componentes presentes no cigarro podem levar a uma deficiência nos níveis de estrógeno, contribuindo para o aparecimento de IU, entretanto no presente estudo nenhuma das participantes relatou ser tabagista (HIGA; LOPES; REIS, 2008).

Em relação ao ICIQ-SF 63 das 79 acadêmicas entrevistadas nunca perderam urina. Das 16 incontinentes 8 perdiam urina uma vez por semana ou menos; 10 acreditam que perdiam uma pequena quantidade; e a média do escore obtida foi de $1,39 \pm 3,23$. No que diz respeito às situações em que perdem urina, 6 participantes relataram que ocorre antes de chegar ao banheiro, 6 durante tosse ou espirro, 2 sem razão óbvia, 1 quando está dormindo e/ou praticando exercícios e 1 durante tosse ou espirro e/ou quando terminou de urinar (Tabela 2).

Padilha et al. (2018) explicitam que o ICIQ-SF é um instrumento usado para verificar a presença de IU sendo considerado simples, breve e autoaplicável. Carvalho et al. (2014), Sousa e Silva (2018) e Cavalcante et al. (2014), utilizaram esse questionário para avaliar a existência de IU na população idosa. Porém, no presente estudo, o ICIQ-SF foi aplicado à população mais jovem, sendo a média de idade de 22,08 anos. Assim, como relatado anteriormente a IU também pode afetar esta população, conforme demonstram os resultados obtidos nesta análise, em que dezesseis $(20,3 \%)$ participantes afirmaram perder urina.

$\mathrm{Na}$ amostra estudada, 8 relataram perder urina uma vez por semana ou menos; 6 diversas vezes ao dia; 1 afirmou perder duas ou três vezes por semana; apenas 1 informou que somente uma vez ao dia; e as outras 63 nunca perderam urina. Com relação à quantidade de urina perdida, 10 das 16 acadêmicas incontinentes relataram que perdem uma pequena quantidade e as outras 6 uma quantidade moderada. A quantidade perdida referida por cada indivíduo é subjetiva e também depende da quantidade de líquidos ingeridos, que podem levar ao aumento da produção de urina (OLIVEIRA et al., 2009). 
Tabela 2 - Resultados dos itens avaliados no ICIQ-SF

\begin{tabular}{|c|c|c|}
\hline Itens & $\mathbf{N}$ & $\%$ \\
\hline \multicolumn{3}{|l|}{ Frequência com que perde urina } \\
\hline Nunca & 63 & 79,7 \\
\hline Uma vez ou menos por semana & 8 & 10,1 \\
\hline Duas ou três vezes por semana & 1 & 1,3 \\
\hline Uma vez ao dia & 1 & 1,3 \\
\hline Diversas vezes ao dia & 6 & 7,6 \\
\hline O tempo todo & 0 & 0 \\
\hline \multicolumn{3}{|l|}{ Quantidade de urina que perde } \\
\hline Nenhuma & 63 & 79,7 \\
\hline Pequena & 10 & 12,7 \\
\hline Moderada & 6 & 7,6 \\
\hline Grande & 0 & 0 \\
\hline \multicolumn{3}{|l|}{ Quando perde urina } \\
\hline Nunca & 63 & 79,7 \\
\hline Antes de chegar ao banheiro & 6 & 7,6 \\
\hline Tosse ou espirro & 6 & 7,6 \\
\hline Quando está dormindo e fazendo atividades físicas & 1 & 1,3 \\
\hline Tosse ou espirro e quando terminou de urinar e está se vestindo & 1 & 1,3 \\
\hline Sem razão óbvia & 2 & 2,5 \\
\hline O tempo todo & 0 & 0 \\
\hline Interferência na vida diária & \multicolumn{2}{|c|}{$0,36 \pm 1,2 *$} \\
\hline ICIQ-SF Escore & \multicolumn{2}{|c|}{$1,39 \pm 3,23^{*}$} \\
\hline
\end{tabular}

Nota: *média; \pm , desvio-padrão.

Fonte: os autores.

O impacto da IU na vida diária foi quantificado pelas respostas das questões 3 , 4 e 5 do ICIQ-SF. A média do escore foi de 1,39 que, de acordo com Silva e D'elboux (2012), é considerado impacto leve. Na acepção de Silva e Lopes (2009), muitas mulheres pensam que a IU é algo normal ou não consideram como um problema importante. Além disso, para Cruz, Collet e Nóbrega (2018) a percepção do indivíduo acerca da sua qualidade de vida, segundo a OMS, varia conforme o contexto no qual está inserido, o sistema de valores nos quais acredita e em relação aos seus padrões e preocupações.

Carvalho et al. (2014) apontam que o ICIQ - SF engloba um conjunto de oito itens de auto diagnóstico relacionados às causas ou situações em que ocorrem as perdas, permitindo assim a classificação quanto ao tipo de IU. No entanto, no presente estudo não foram definidos os tipos, pois de acordo com Almeida e Machado (2012), é necessária uma avaliação clínica, além de exames que complementem o diagnóstico e que auxiliem na classificação das perdas.

Para verificar a associação entre os fatores de risco e a IU, presente nas participantes, foi aplicado o teste de Qui-quadrado, sendo adotado o valor $\mathrm{p}<0,05$ (Tabela 3).

A partir dos resultados obtidos apenas a infecção urinária $(\mathrm{p}=0,020)$ teve correlação com a perda involuntária de urina. No presente estudo 13 das 16 incontinentes relataram um ou mais episódios de infecção urinária, totalizando $81,25 \%$, fator este que pode ter influenciado na perda de urina.

De acordo com Baracho (2002) e Girão, Lima e Baracat (2009) o histórico recorrente de infecção urinária pode levar a IU devido à redução da pressão de fechamento uretral. Além disso, este quadro patológico pode levar a sintomas como aumento da frequência urinária e urgência miccional. 
Tabela 3 - Associação entre as variáveis: fatores de risco e incontinência urinária

\begin{tabular}{lc}
\hline Fatores de risco & Incontinência urinária (valor de p) \\
\hline Idade & 0,605 \\
IMC & 0,750 \\
Atividade Física & 0,490 \\
Infecção urinária & $0,020^{*}$ \\
\hline Ingestão de líquidos & \\
\hline Café & 0,455 \\
Chá & 0,696 \\
Sucos & 0,540 \\
Refrigerante & 0,970 \\
Bebidas alcoólicas & 0,865 \\
\hline Menstruação & \\
\hline Irregular & 0,300 \\
Não menstrua mais de um ano & 0,301 \\
\hline Tipo de parto & \\
\hline Vaginal & 0,612 \\
Cesárea & 0,988 \\
\hline Etnia & 0,150 \\
\hline HAS & 0,612 \\
\hline NÁt
\end{tabular}

Nota:*valor de significância $(\mathrm{p}<0,05)$

Fonte: os autores.

\section{CONCLUSÃO}

Com base nos resultados deste estudo, concluiu-se que apenas uma parcela das acadêmicas apresentou incontinência urinária e, entre os fatores de risco avaliados, somente a infecção urinária evidenciou associação com a perda involuntária de urina.

Em relação ao perfil da amostra estudada, a maioria era de etnia branca, com média de idade de 22,08 anos, nulíparas e sedentárias. Foi encontrada uma prevalência de 20,3\% de casos de incontinência urinária e dentre os fatores de risco avaliados apenas a infecção urinária apresentou associação com perda involuntária de urina.

Dessa forma, sugere-se que sejam realizadas novas pesquisas que abordem a mesma temática, com outras formas de avaliação que possam complementar esses achados e que também utilizem a faixa etária mais jovem, pois, grande parte dos estudos presentes na literatura abrange a população idosa.

Ressalta-se como limitação deste estudo a possível falta de interesse ou de conhecimento sobre o tema, visto que mais de $20 \%$ dos questionários foram excluídos por estarem preenchidos incorretamente.

\section{REFERÊNCIAS}

ALMEIDA, P. P.; MACHADO, L. R. G. A prevalência de incontinência urinária em mulheres praticantes de jump. Fisioterapia em movimento, v. 25, n. 1, p. 55-65, 2012. Disponível em: http://www.scielo.br/scielo.php?script=sci_arttext\& pid=S0103-51502012000100006. Acesso em: 26 jul. 2019.

BARACHO, E. Fisioterapia aplicada à obstetrícia: aspectos de ginecologia e neonatologia. 3 . ed. Rio de Janeiro: Medsi, 2002. 
BEREK, J. S. Berek \& Novak: Tratado de Ginecologia. 14. ed. Rio de Janeiro: Guanabara Koogan, 2012.

BRASIL. Ministério da Saúde. IMC em adultos. 2017. Disponível em: http://www.saude.gov.br/component/content/article/804-imc/40509-imc-em-adultos. Acesso em: 19 jul. 2019.

CÂNDIDO, F. J. L. F. et al. Incontinência urinária em mulheres: breve revisão de fisiopatologia, avaliação e tratamento. Visão Acadêmica, v. 18, n. 3, 2017. Disponível em: https://revistas.ufpr.br/academica/article/view/54506. Acesso em: 12 ago. 2019.

CARVALHO, M. P. et al. O impacto da incontinência urinária e seus fatores associados em idosas. Revista Brasileira de Geriatria e Gerontologia, v. 17, n. 4, p. 721-730, 2014. Disponível em: http://www.scielo.br/pdf/rbgg/v17n4/1809-9823-rbgg-17-04-00721.pdf. Acesso em: 14 jul. 2019.

CAVALCANTE, K. V. M. et al. Prevalência e fatores associados à incontinência urinária em mulheres idosas. Revista Brasileira em Promoção da Saúde, v. 27. n. 2, p. 216-223, 2014. Disponível em: https://periodicos.unifor.br/RBPS/article/view/2528. Acesso em: 6 ago. 2019.

CRUZ, D. S. M.; COLLET, N.; NÓBREGA, V. M. Qualidade de vida relacionada à saúde de adolescentes com dm1 - revisão integrativa. Ciência \& Saúde Coletiva, v. 23, n. 3, p. 973-989, 2018. Disponível em: http://www.scielo.br/scielo.php?script=sci_arttext\&pid=S141381232018000300973. Acesso em: 6 ago. 2019.

FORTUNATO, G. L. et al. Correlação entre a força dos músculos do assoalho pélvico e a satisfação sexual de mulheres. Cadernos da Escola de Saúde, v. 2, n. 6, p. 143-158, 2014. Disponível em: http://portaldeperiodicos.unibrasil.com.br/index.php/cadernossaude/article/view/2336. Acesso em: 19 fev. 2019.

GASPARETTO, A. et al. Efeitos da fisioterapia com abordagem em grupo sobre a incontinência urinária feminina na atenção primária de saúde em Santa Maria. Disciplinarum Scientia. Série: ciências da saúde, v. 12, n. 1, p. 59-70, 2011. Disponível em: https://periodicos.ufn.edu.br/index. php/disciplinarumS/article/view/977. Acesso em: 25 jul. 2019.

GIRÃO, M. J. B. C.; LIMA, G. R.; BARACT, E. C. Ginecologia. São Paulo: Manole, 2009.

GROSSE, D.; SENGLER, J. Reeducação Perineal. São Paulo: Manole, 2002.

HIGA, R.; LOPES, M. H. B. M.; REIS, M. J. Fatores de risco para incontinência urinária na mulher. Revista da Escola de Enfermagem da USP, v. 42, n. 1, p. 187-192, 2008. Disponível em: http://www.scielo.br/scielo.php?pid=s0080-62342008000100025\&script=sci_abstract\&tlng=pt. Acesso em: 8 ago. 2018.

JARDIM, A. D. I. et al. Hipertensão arterial e incontinência urinária no idoso: revisão integrativa da literatura. CuidArte Enfermagem, v. 5, n. 1, p. 38-43, 2011. Disponível em: http://bases.bireme.br/cgi-bin/wxislind.exe/iah/online/?IsisScript=iah/iah.xis\&src=google\&base= BDENF\&lang=p\&nextAction=lnk\&exprSearch=20573\&indexSearch=ID. Acesso em: 25 jul. 2019.

JUSTINA, L. B. D. Prevalência de incontinência urinária feminina no Brasil: uma revisão sistemática. Revista Inspirar - Movimento \& Saúde, v. 6, n. 3, 2013. Disponível em: 
https://www.inspirar.com.br/wp-content/uploads/2014/10/prevalencia-de-incontinencia-artigo313.pdf. Acesso em: 6 ago. 2018.

LEROY, L. S.; LOPES, M. H. B. M.; SHIMO, A. K. K. A incontinência urinária em mulheres e os aspectos raciais: uma revisão de literatura. Texto \& Contexto - Enfermagem, v. 21, n. 3, p. 692701, 2012. Disponível em: http://www.scielo.br/scielo.php?pid=S0104-07072012000300026\&script =sci_abstract\&tlng=pt. Acesso em: 14 jul. 2019.

MCANINCH, J. W.; LUE, T. F. Urologia geral de Smith e Tanagho. 18. ed. Porto Alegre: AMGH, 2014.

MONTELLATO, N.; BARACAT, F.; ARAP, S. Uroginecologia. São Paulo: Roca, 2000.

MOURÃO, L. F. et al. Caracterização e fatores de risco de incontinência urinária em mulheres atendidas em uma clínica ginecológica. Estima, v. 15, n. 2, p. 82-91, 2017. Disponível em: https://www.revistaestima.com.br/index.php/estima/article/view/352. Acesso em: 7 ago. 2018.

OLIVEIRA, S. G. et al. Avaliação da qualidade de vida de portadores de incontinência urinária. Revista Brasileira de Ciências do Envelhecimento Humano, v. 6, n. 1, p. 34-41, 2009. Disponível em: http://seer.upf.br/index.php/rbceh/article/view/251. Acesso em: 29 jul. 2019.

PADILHA, J. F. et al. Investigação da qualidade de vida de mulheres com incontinência urinária. Arquivos de Ciências da Saúde UNIPAR, v. 22, n. 1, p. 43-48, 2018. Disponível em: http://revistas.unipar.br/index.php/saude/article/view/6302. Acesso em: 29 jul. 2019.

PATRIZZI, L. J. et al. Incontinência urinária em mulheres jovens praticantes de exercício físico. Revista Brasileira de Ciência e Movimento, v. 22, n. 3, p. 105-110, 2014. Disponível em: https://portalrevistas.ucb.br/index.php/RBCM/article/view/4185. Acesso em: 6 jul. 2019.

POLDEN, M.; MANTLE, J. Fisioterapia em Ginecologia e Obstetrícia. 2. ed. São Paulo: Santos, 2002.

SANTOS, E. S. et al. Incontinência urinária entre estudantes de educação física. Revista da Escola de Enfermagem da USP, v. 43, n. 2, p. 307-312, 2009. Disponível em: http://www.scielo.br/scielo.php?script=sci_arttext\&pid=S0080-62342009000200008. Acesso em: 8 ago. 2018.

SILVA, L.; LOPES, M. H. B. M. Incontinência urinária em mulheres: razões da não procura por tratamento. Revista da Escola de Enfermagem da USP, v. 43, n. 1, p. 72-78, 2009. Disponível em: http://www.scielo.br/scielo.php?script=sci_arttext\&pid=S0080-62342009000100009. Acesso em: 4 ago. 2019.

SILVA, V. A.; D’ELBOUX, M. J. Fatores associados à incontinência urinária em idosos com critérios de fragilidade. Texto Contexto Enfermagem, v. 21, n. 2, p. 338-347, 2012. Disponível em: http://www.scielo.br/scielo.php?pid=S0104-07072012000200011\&script=sciabstract\&tlng=pt. Acesso em: 19 jun. 2019.

SOUSA, M. P. S.; SILVA, J. M. Prevalência de incontinência urinária em idosas. Revista Saúde em foco, v. 5, n. 2, p. 40-49, 2018. Disponível em: http://www4.fsanet.com.br/revista/index.php/ saudeemfoco/article/view/1720. Acesso em: 29 jul. 2019. 
TAMANINI, J. T. N. et al. Validação para o português do "International Consultation on Incontinence Questionnaire - Short Form" (ICIQ - SF). Revista Saúde Pública, v. 38, n. 3, p. 438444, 2004. Disponível em: http://www.scielo.br/scielo.php?script=sci_arttext\&pid=S003489102004000300015. Acesso em: 26 jul. 2019.

TETTAMANTI, G. et al. Effects of coffee and tea consumption on urinary incontinence in female twins. BJOG. v. 118, n. 7, p. 806-813, 2011. Disponível em: https://obgyn.onlinelibrary.wiley.com/ doi/full/10.1111/j.1471-0528.2011.02930.x. Acesso em: 16 out. 2019.

VIRTUOSO, J. F.; MAZO, G. Z.; MENEZES, E. C. Incontinência Urinária e função muscular perineal em idosas praticantes e não-praticantes de atividade física regular. Revista Brasileira de Fisioterapia, v. 15, n. 4, p. 310-317, 2011. Disponível em: http://www.scielo.br/scielo.php?script= sci_arttext\&pid=S1413-35552011000400009\&lng=en\&nrm=iso\&tlng=pt. Acesso em: 10 ago. 2019. 